\title{
Hyalinizing trabecular tumor of the thyroid gland
}

\begin{abstract}
Sefa Ergün ${ }^{1}$ (D), Ozan AkıncI' Tülin Öztürk², Adem Karataș $^{1}$
Hyalinizing trabecular tumor was first described by Carney et al. (1) in 1987 and is a rare benign tumor of the thyroid gland that shares some of the microscopic features of medullary and papillary thyroid carcinoma. Hyalinizing trabecular tumor derives from follicular cells, and it is characterized by an apparent trabecular pattern and intratrabecular hyalinization. In this study, we present the case of a 40 -year-old female patient with thyroid gland nodules, whose ultrasound results, clinical behavior, and fine-needle aspiration biopsy results were suspicious; the pathology after thyroidectomy indicated hyalinizing trabecular tumor. We aimed to show the role of clinical behavior, radiology, fine-needle aspiration, and histological and immunohistochemical analysis in the differential diagnosis of hyalinizing trabecular tumor. Hyalinizing trabecular tumor which can be confused with papillary and medullar carcinoma of the thyroid gland, is mostly benign but some malignant and metastatic cases have been reported. Therefore, diagnosis, treatment, and follow-up steps of Hyalinizing trabecular tumor should be planned in consideration of a malignant potential.
\end{abstract}

ORCID ID of the corresponding author: S.E 0000-0002-0315-8044

Cite this paper as: Ergün S, Akıncı 0, Öztürk T, Karataş A. Hyalinizing trabecular tumor of the thyroid gland. Turk J Surg 2018; 34: 149-151.

'Department of General Surgery, İstanbul University Cerrahpaşa School of Medicine, İstanbul, Turkey

2Department of Clinical Pathology, İstanbul University Cerrahpaşa School of Medicine, İstanbul, Turkey

\section{Corresponding Author Sefa Ergün}

e-mail: sefaergn@yahoo.com

Received: 28.05.2015

Accepted: 10.08.2015

Available Online Date: 03.01.2018

oCopyright 2018

by Turkish Surgical Association

Available online at

www.turkjsurg.com
Keywords: Thyroid tumors, hyalinizing trabecular tumor, trabecular pattern

\section{INTRODUCTION}

Hyalinizing trabecular tumor (HTT) is a rare thyroid gland neoplasm that was first described in 1987 by Carney et al. (1). It is a thyroid tumor of a follicular origin and is characterized by trabecular pattern and intratrabecular hyalinization (2). HTT entered World Health Organization's (WHO) Thyroid Tumor Classification in 2004 (3). Cytological appearance of HTT is mostly confused with malignant lesions that need thyroidectomy and lymph node dissection. Several cytological and histologic features of HTT are identical to those of papillary thyroid carcinoma (PTC) and medullary thyroid carcinoma. Some authors suggest that HTT is not a different type of thyroid carcinoma, but rather a variant of PTC. Preoperative cytological diagnosis is rarely accurate (4). Most cases are benign in nature; however, some HTT cases have been reported with lung and lymph node metastasis (5). Furthermore, microscopic appearance of HTT is similar to that of paraganglioma (5).

In this article, we will present the clinical and pathological features of HTT and focus on the differential diagnosis of a female patient who was recognized to have HTT after thyroidectomy.

\section{CASE PRESENTATION}

A 40-year-old female patient was admitted with difficulty in swallowing. The patient's thyroid ultrasound (US) result demonstrated a $15 \times 9 \mathrm{~mm}$ heterogeneous hypoechoic solid nodule on right lobe of the thyroid gland. Fine-needle aspiration biopsy (FNAB) was performed. Cytological examination showed atypical epithelial cells with hypercellular and trabecular patterns around the nucleus and fine chromatin, and some cells had grooves. Result was inconclusive for occurrences of papillary carcinoma and medullary carcinoma.

Magnetic resonance imaging (MRI) results demonstrated a $12 \times 10 \mathrm{~mm}$ T1A hypointense, T2A hypointense, well circumscribed nodule on the right lobe of the thyroid gland. For differential diagnosis of medullary carcinoma, MRI of the pituitary gland was performed and no pathological result was obtained. Patient was euthyroid. Free thyroxin (fT4) and thyroid stimulating hormone (TSH) levels were in normal range. Calcitonin value was normal for a female. Operation was decided on for the patient, and total thyroidectomy was performed. Postoperative pathological diagnosis was HTT of the thyroid gland.

Macroscopic examination on thyroidectomy: $1.2-\mathrm{cm}$ diameter well circumscribed, regular, homogenous, yellow-colored, nodular lesion under the capsule of the right thyroid lobe. Results of the microscopic examination: the nodule forms a clear trabecular pattern and has extended polygonal cells that include the nuclear notch with pseudoinclusions in some places (Figure 1). There was stromal hya- 


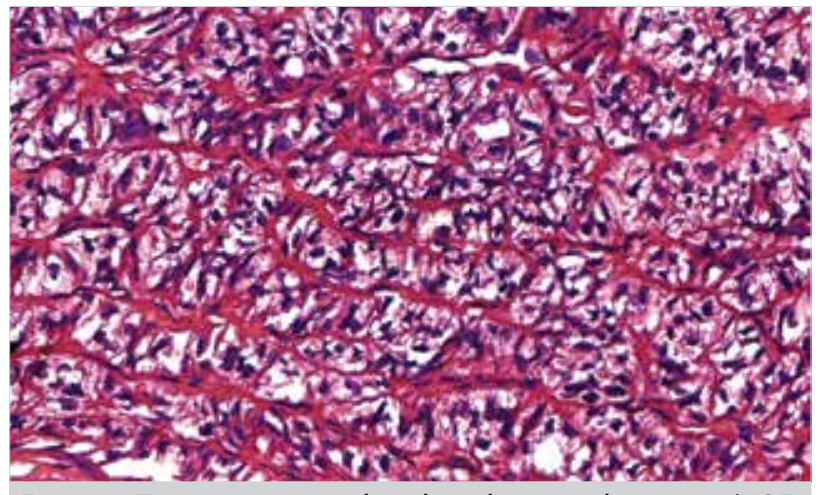

Figure 1. Tumor tissues with trabecular growth pattern (H\&E; $400 \times)$

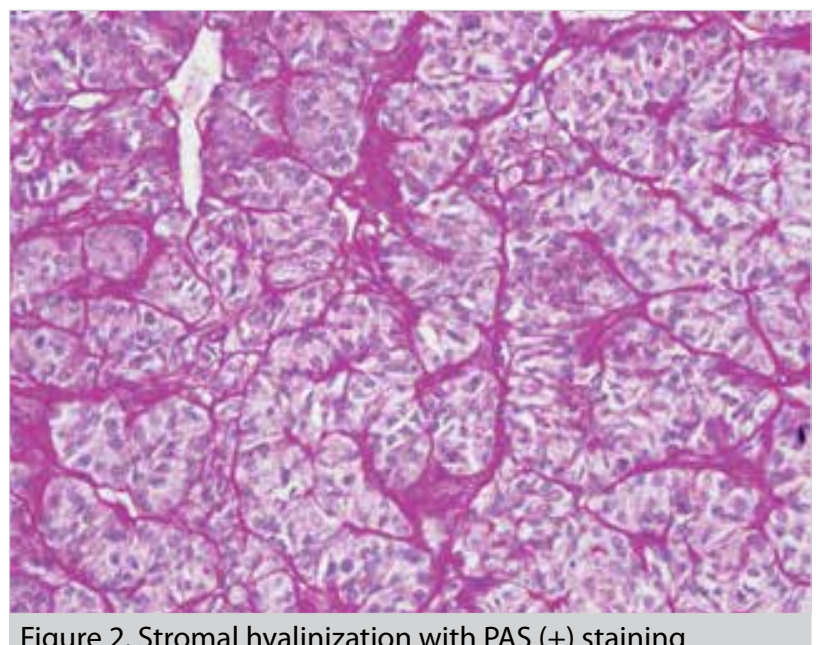

Figure 2. Stromal hyalinization with PAS (+) staining

linization between trabeculae. On histochemical examination, these hyalinized areas stained positive for Periodic acid-Schiff (PAS) (Figure 2). Furthermore, nonspecific lymphocytic thyroiditis was seen around the nodule.

On immunohistochemical analysis, cells that form the nodules stained positive for thyroglobulin antibody; there was no staining for chromogranin, calcitonin, carcinoembryonic antigen (CEA), and cytokeratin (CK) 19. Hector Battifora Mesothelial 1 (HBME-1) was found to be focal weak positive.

No complications occurred after the surgery. The patient was discharged on the first postoperative day. L-Thyroxine treatment was initiated. Control examination on cervical US was performed 1-year postoperation. No residual tissue or metastatic lesions were detected. A written informed consent was received from the patient.

\section{DISCUSSION}

According to World Health Organization's (WHO) classification of Endocrine Organ Tumors, HTT is described as "an uncommon tumor of follicular origin characterized by trabecular pattern of growth and marked intra-trabecular hyalinization (3). HTT is a well-circumscribed, encapsulated lesion and usually asymptomatic; it can be seen in forms of single or numerous nodules or incidentally after thyroidectomy. Mostly it is common in middle-aged women; female:male ratio is 6:1. Rarely, HTTs are associated with chronic lymphocytic thyroiditis or
The basic histological structure of HTT is characterized with a hyalinizing trabecular pattern, cytoplasmic inclusions at cell cytoplasm and matrix, and nucleus with grooves (5). These tumors have the same macroscopic appearance as the classical thyroid adenomas. Their special features are perivascular hyalinization, dominant stroma, and trabecular arrangement. Organoid strictures are similar to papillary carcinoma $(5,6)$.

Another controversial point on HTT is the benign or malignant status of the tumor. In 1987, when Carney et al. (1) first described the tumor, they said that HTT is a benign lesion and does not cause to vascular invasion or metastasis. After that, HTT was claimed to be a benign lesion in many cases; however, the literature shows malignant forms that cause capsule and vessel invasion or lung and lymph node metastasis (5). In our case, the tumor was well-circumscribed and had no invasion to vessels and surrounding thyroid tissue.

Macroscopically, these tumors are well-circumscribed and encapsulated like other adenomas. Their most distinctive microscopic features are hyalinized stroma-like amyloid and trabecular pattern of tumor cells (1). Due to the organoid structures accompanying this trabecular pattern, they are called "paraganglioma-like adenoma".

Histologically, the detection of intranuclear groove, pseudoinclusions, and psammoma bodies cause confusion with PTC (7). Further, the fact that HTT is accompanied by papillary carcinoma and that they have the same immunophenotypic properties cause interpretations that both tumors are similar, and moreover, that $\mathrm{HTT}$ is a special variant of papillary carcinomas. In our case, FNAB raised a suspicion of PTC; however, pathological study of the material showed no PTC near HTT.

Hyalinizing trabecular tumor can be confused with encapsulated variant of medullary thyroid carcinoma. The base of this confusion is the presence of spindle cells and intratrabecular hyalinization that looks like stromal amyloid (8). These tumors can be distinguished from medullary carcinoma as they are negative for Congo red stain, positive for thyroglobulin, and negative for calcitonin(8). In our case, immunohistochemical analysis was negative for calcitonin.

Hector Battifora Mesothelial 1 is a monoclonal antibody found in mice that is used as a marker for malignant mesothelioma cells. Its sensitivity is $80 \%-90 \%$ and specificity is between $60 \%$ and $96 \%$ for cancers originating from follicular cells. Mostly it is negative for medullar and undifferentiated carcinoma (9). In our case, immunohistochemical analysis for HBME-1 was focal weak positive.

\section{CONCLUSION}

Because of biological and clinical behavior, HTT should be approached as a benign neoplasm; however, although low, its malignant potentiall should not be overlooked $(5,10)$. It is mostly confused with PTC, but in differential diagnosis, medullary thyroid carcinoma, paraganglioma, and follicular adenoma should be taken into consideration as well.

Treatment of HTT is conservative like PTC, and surgery is performed under similar terms as those for PTC. In the postop- 
erative period, HTT should be follow up like a malignant neoplasm (10). Wider case studies with suitable follow-ups will be helpful in explaining HTT development and behavior.

Informed Consent: Written informed consent was obtained from patient who participated in this study.

Peer-review: Externally peer-reviewed.

Author Contributions: Concept -S.E., A.K.; Design - S.E., O.A.; Supervision - A.K., T.Ö.; Resource - S.E., O.A.; Materials - S.E., O.A., T.Ö.; Data Collection and/or Processing - S.E., O.A., T.Ö.; Analysis and/or Interpretation - T.Ö., A.K.; Literature Search - S.E., O.A.; Writing Manuscript - S.E., O.A.; Critical Reviews - T.Ö., A.K.

Conflict of Interest: The authors have no conflicts of interest to declare.

Financial Disclosure: The authors declared that this study has received no financial support.

\section{REFERENCES}

1. Carney JA, Ryan J, Goellner JR. Hyalinizing trabecular adenoma of the thyroid gland. Am J Surg Pathol 1987; 11: 583-591. [CrossRef]

2. Ertan Y, Makay Ö, Veral A, Tamsel S, Içöz G, Tunçyürek M. Hyalinizing trabecular tumor of the thyroid. Turk Patoloji Derg 2009; 25: 44-46. [CrossRef]
3. DeLellis RA, Lloyd RV, Heitz PU, Eng C (Eds): World Health Organization Classification of Tumours. Pathology and Genetics of Tumours of Endocrine Organs. Lyon, IARC Pres, 2004.

4. Lester DR. Thompson MD. Hyalinizing trabecular adenoma of the thyroid gland. Ear Nose Throat J 2011; 90: 416-417.

5. Gowrishankar S, Pai SA, Carney JA. Hyalinizing trabecular carcinoma of the thyroid gland. Histopathology 2008; 52: 529531. [CrossRef]

6. Nose ' V, Volante M, Papotti M. Hyalinizing trabecular tumor of the thyroid: an update. Endocr Pathol 2008; 19: 1-8. [CrossRef]

7. Fonseca E, Nesland JM, Sobrinho-Simões M. Expression of stratified epithelial-type cytokeratins in hyalinizing trabecular adenomas supports their relationship with papillary carcinomas of the thyroid. Histopathology 1997; 31: 330-335. [CrossRef]

8. Katoh R, Jasani B, Williams ED. Hyalinizing trabecular adenoma of the thyroid. A report of three cases with immunohistochemical and ultrastructural studies. Histopathology 1989; 15: 211 224. [CrossRef]

9. De Micco C, Savchenko V, Giorgi R, Sebag F, Henry JF. Utility of malignancy markers in fine-needle aspiration cytology of thyroid nodules: comparison of Hector Battifora mesothelial antigen-1, thyroid peroxidase and dipeptidyl aminopeptidase IV Br J Cancer 2008; 98: 818-823. [CrossRef]

10. Carney JA, Hirokawa M, Lloyd RV, Papotti M, Sebo TJ Hyalinizing trabecular tumors of the thyroid gland are almost all benign. Am J Surg Pathol 2008; 32: 1877-1889. [CrossRef] 